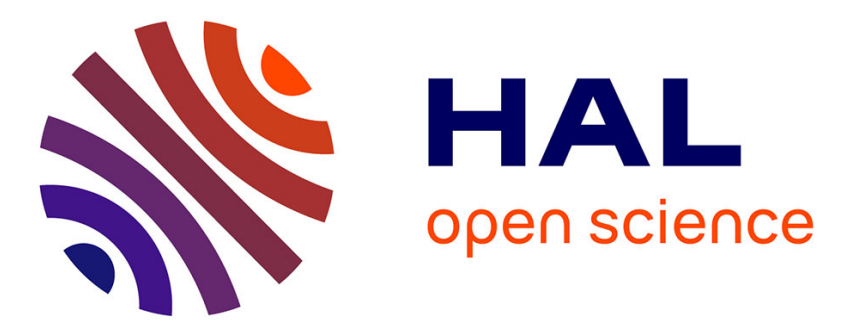

\title{
Simplified model enabling optimization of silicon modulators
}

Diego Perez-Galacho, Delphine Marris-Morini, Remco Stoffer, Eric Cassan, Charles Baudot, Twan Korthorst, Frederic Boeuf, Laurent Vivien

\section{- To cite this version:}

Diego Perez-Galacho, Delphine Marris-Morini, Remco Stoffer, Eric Cassan, Charles Baudot, et al.. Simplified model enabling optimization of silicon modulators. SPIE Optics and Optoelectronics, Apr 2017, Prague Czech Republic. 10.1117/12.2265792 . hal-01544720

\author{
HAL Id: hal-01544720 \\ https://hal.science/hal-01544720
}

Submitted on 22 Jun 2017

HAL is a multi-disciplinary open access archive for the deposit and dissemination of scientific research documents, whether they are published or not. The documents may come from teaching and research institutions in France or abroad, or from public or private research centers.
L'archive ouverte pluridisciplinaire HAL, est destinée au dépôt et à la diffusion de documents scientifiques de niveau recherche, publiés ou non, émanant des établissements d'enseignement et de recherche français ou étrangers, des laboratoires publics ou privés. 


\title{
Simplified model enabling optimization of silicon modulators
}

\author{
Diego Perez-Galacho ${ }^{\mathrm{a}}$, Delphine Marris-Morini ${ }^{\mathrm{a}}$, Remco Stoffer ${ }^{\mathrm{b}}$, Eric Cassan ${ }^{\mathrm{a}}$, Charles \\ Baudot $^{\mathrm{c}}$, Twan Korthorst ${ }^{\mathrm{b}}$, Frederic Boeuf ${ }^{\mathrm{c}}$, and Laurent Vivien ${ }^{\mathrm{b}}$ \\ ${ }^{a}$ Center for Nanoscience and Nanotechnology, CNRS, Univ. Paris-Sud, Universite \\ Paris-Saclay,C2N-Orsay, 91405 Orsay Cedex, France \\ ${ }^{b}$ PhoeniX BV, Hengelosestraat 705, 7521 PA Enschede, The Netherlands \\ ${ }^{\mathrm{c} S T}$ Microelectronics, 850 rue Jean Monnet 38920 Crolles, France
}

\begin{abstract}
In this work, the simplified modeling of silicon phase modulators is presented along with a comparison among different options of modulators. The proposed simplified model enables a substantial reduce in computational effort while maintaining a good accuracy. The presented model is validated against complete 3D-simulations by means of the design of four different modulators. Furthermore, with the help of the model a deep insight on the performances tradeoffs in the choose and design of silicon modulators is provided.
\end{abstract}

Keywords: Modulators, Silicon photonics, Optoelectronics, Integrated Optics, Simulation

\section{INTRODUCTION}

Nowadays, Silicon photonics has became a reference platform for many photonics based applications, such as sensing or telecommunication. It enables the integration with current microtechnology circuitry thanks to its CMOS compatibility. Due to that, it is expected that silicon photonics will play a key role in next generation photonics technologies, like intra- and inter-chip optical interconnets. ${ }^{1}$ Silicon modulators are very important in many photonics applications. ${ }^{2}$ Some examples are: sensors based on heterodyne detection, ${ }^{3}$ transmitters ${ }^{4-6}$ and coherent beam combining systems. ${ }^{7,8}$ Modulators specifications greatly vary on the targeted application. Bandwidth is normally the critical parameter in data communication. Low loss and high efficiency, however, are normally required in sensing applications. Chirp is also important sometimes. In-depth understanding of the relationships among the performance and the modulator design parameters is a must to properly choose a modulator for a given application. Moreover, simplified modelling of modulators plays a key role in the modulator design stage and as a tool to understand modulators intrinsic performance tradeoffs. In this work simplified modelling of silicon phase modulator is derived and used to present the performance tradeoffs in the design of silicon modulators. The presented models are evaluated and validated against complete 3D simulations. Furthermore, models are used to design four different modulators and a discussion comparing them is provided.

\section{SIMPLIFIED MODELING OF PHASE MODULATORS}

In order to model silicon phase modulators based on Free-Carrier Plasma Dispersion Effect (FCPD) it is necessary to model the optical aspect of the modulator (waveguide mode), the electrical aspect (carriers distribution) and the interaction between them. A thorough study on this interaction was carried out by Soref et al. in. ${ }^{9}$ As a result empirical equations relating carriers distributions and refractive index changes were provided. Soref's equations

\footnotetext{
Further author information: (Send correspondence to D. Perez-Galacho)

D. Perez-Galacho.: E-mail: diego.perez-galacho@u-psud.fr, Telephone: +33 (0) 169153482

D. Marris-Morini: E-mail: delphine.morini@u-psud.fr, Telephone: +33 (0) 169157852

R. Stoffer: E-mail: remco.stoffer@phoenixbv.com, Telephone: +31 (0)534836460

E. Cassan: E-mail: eric.cassan@u-psud.fr, Telephone: +33 (0) 169157852

C. Baudot: E-mail: charles.baudot@st.com

T. Korthorst: E-mail: twan.korthorst@phoenixbv.com, Telephone: +31 (0)534836460

F. Boeuf: E-mail: frederic.boeuf@st.com

L. Vivien: E-mail: laurent.vivien@u-psud.fr, Telephone: +33 (0) 169154070
} 
are used hereafter to model the interaction between the electrical and the optical parts of the modulators. In Fig. 1 (a) the general flux diagram of the complete simulation is represented, this is the approach generally used to analyze silicon modulators. For each set of modulator parameters it involves a complex 2D or 3D electrical simulation and a precise mode simulation for each point of the electrical simulation. It is important to point out that to properly take into account the effect of carriers the modal simulation should be done with a solver based on Finite Element or Finite Difference discretization schemes with a really fine mesh $(\sim 5 \mathrm{~nm})$, requiring large simulation times, thus one modal simulation should be carried out for every simulated voltage. This hinders the design and the optimization possibilities of phase modulators. However, the use of a simplified model for phase modulators permits reduced memory requirements and simulation time, maintaining a fairly good level of accuracy at the same time. The model schematized in Fig. 1 (b) is proposed to fulfill these requirements. Firstly the mode of the waveguide is calculated considering it passive i.e. there is no carriers in it. This is represented in Fig. 1 (b) as the box "Modal Simulation". The results of this box are the electric field $(E(x, y))$ and the effective index $\left(\mathrm{n}_{\text {eff }}\right)$ of the modes. Secondly, a 1D simulation is used to model the electrical part, with the exception of the reverse biased PN junction that can be modeled analytically. Carrier distributions along the junction direction are the results of the 1D simulation, these carrier distributions are extended in the other two directions of space in order to build a 3D carrier distribution $(n(x, y, z)$ and $p(x, y, z))$. Using the Soref model, these carrier distributions are transformed into refractive index changes $(\Delta \mathrm{n}(x, y, z)$ and $\Delta \mathrm{k}(x, y, z))$. Finally, using perturbation theory, ${ }^{10}$ efficiency an loss are calculated by:

$$
\begin{aligned}
& \Delta \phi=\iiint \Delta \mathrm{n}(x, y, z)|E(x, y)|^{2} d x d y d z \\
& \Delta \alpha=\iiint \Delta \mathrm{k}(x, y, z)|E(x, y)|^{2} d x d y d z
\end{aligned}
$$

where $\Delta \phi$ and $\Delta \alpha$ are the phase shift and the loss change, the mode electric field $(E(x, y))$ is normalized to unity power. The calculation of bandwidth is modeled by the RC equivalent circuit in depletion based modulators, given that it is the main limitation. However, in injection based modulator a transient electrical simulation is necessary to compute the bandwidth. ${ }^{11}$ If we compare both simulation schemes, the complete simulation and the simplified model (see Fig. 1). The first one requires a complex 2D/3D electrical simulation and a mode simulation for each voltage. On the other hand, the second one only a simple 1D electrical simulation (analytical in the case of PN junction), one mode calculation and an overlap integral for each voltage. It is to be expected that the simplified model will drastically reduce the computation effort. Lumerical software suited was used to carry out the electrical and mode simulations. ${ }^{12}$

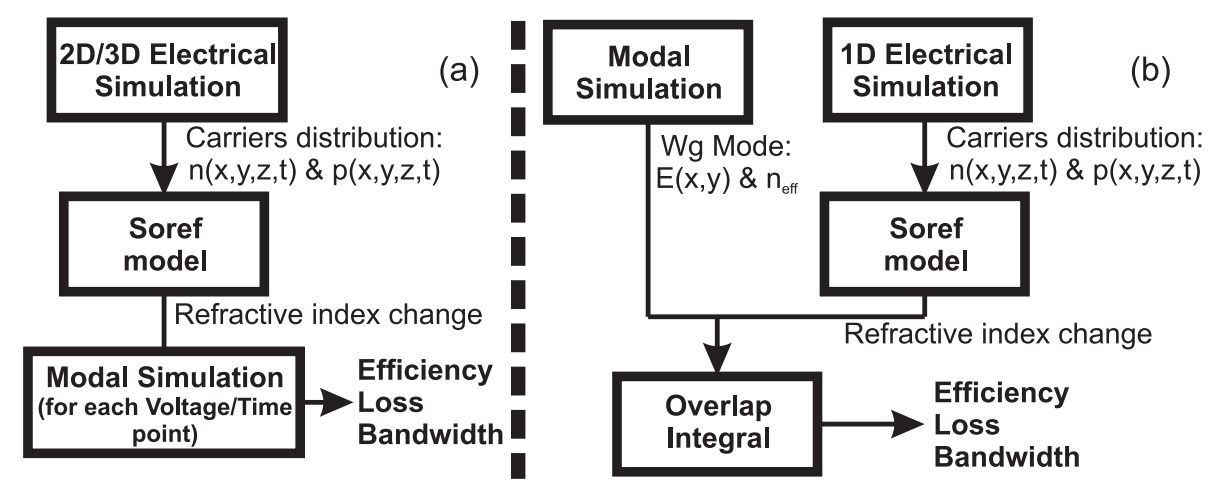

Figure 1. General schematic of the complete simulation (a) and the simplified phase modulator model (b).

\section{MODEL RESULTS AND COMPARISON}

In order to obtain the accuracy of the simplified model and to validate it, four different phase modulators based on carrier depletion and carrier injection were designed using the simplified model. The junctions considered considered were PN, ${ }^{13,14}$ PIPIN, ${ }^{15}$ and PN interdigitated ${ }^{16,17}$ for carrier depletion based modulators and PIN for carrier injection. ${ }^{18}$ Rib waveguides based on $300 \mathrm{~nm}$ height SOI technology were considered, two different 
slab heights $(50 \mathrm{~nm}$ and $150 \mathrm{~nm})$ were analyzed. The design of each modulator was orientated to obtained the best performance according to different specifications. High bandwidth was expected for the PN junction based modulator, the PIPIN junction based modulator was optimized for low loss. The PN interdigitated and the PIN injection were designed to exhibit high efficiency and ultra-high efficiency respectively.

\subsection{High speed PN junction based modulator}

The PN junction based modulator under consideration is sketched in Fig. 2, it was orientated to high speed operation. A $150 \mathrm{~nm}$ slab height waveguide was chosen in order to reduce the access resistance and further optimized bandwidth. In order to maximize the efficiency, the junction position could be displaced from the center of the waveguide.

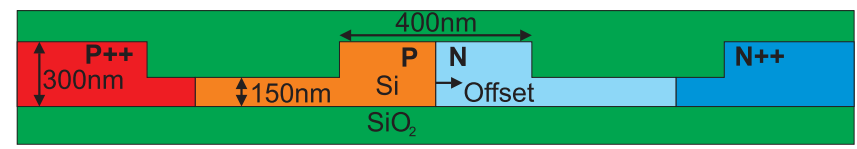

Figure 2. Schematic view of the PN junction based modulator.

The simplified model was used to optimize doping concentrations and junction's offset. This was carried out by sweeping over more than 800 set of parameters, which took less than 4 hours in a desktop computer. The obtained optimized values were $P=N=5 \cdot 10^{17} \mathrm{~cm}^{-3}$ and Offset $=30 \mathrm{~nm}$ for doping concentrations and offset respectively. A complete simulation was carried out with this set of values, which took between 15 and 30 minutes for a single simulation in a high performance computing machine. Results of both the simplified model and the complete simulation are plot in Fig. 3, where efficiency and loss are represented as a function of the applied voltage. Good agreement were obtained between them. The simplified model reported $V_{\pi} L_{\pi}=1.53 \mathrm{Vcm}$ and Loss $=5.3 \mathrm{~dB}$ for an applied voltage of $3 \mathrm{~V}$, while the complete simulation provided $V_{\pi} L_{\pi}=1.51 \mathrm{Vcm}$ and Loss $=4.7 \mathrm{~dB}$. Less than $5 \%$ was obtained using the simplified model. The calculated RC bandwidth of the modulator was over $50 \mathrm{GHz}$ in both cases, enabling high speed operation.
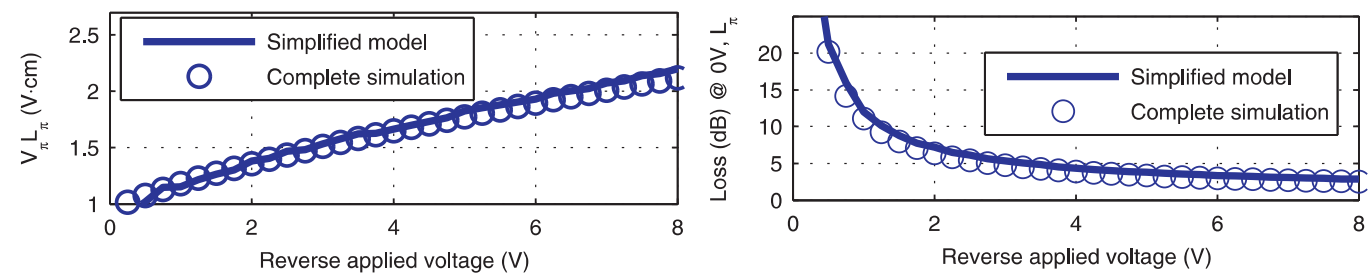

Figure 3. Performance of the PN junction based modulator.

\subsection{Low loss PIPIN junction based modulator}

Reduced loss was the target of the PIPIN junction based modulator. The modulator is represented in Fig. 4, a $\mathrm{P}$ zone is inserted in the middle of a PIN junction reducing the loss while maintaining a fairly good efficiency. The 50nm slab height waveguide was chosen so that optical mode is well confined in the active region and the overlap between the middle $\mathrm{P}$ zone and the optical mode is maximized.

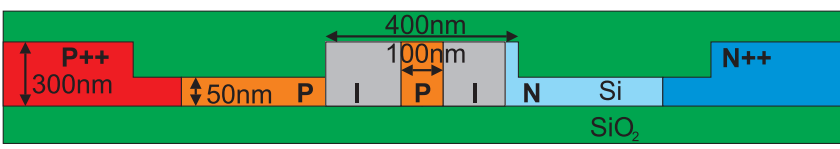

Figure 4. Schematic view of the PIPIN junction based modulator.

Using the simplified model all doping concentrations were optimized, in this case more than 1100 set of values were swept and the computation time was less than 12 hours. It is important to notice that in this case 
a 1 D electrical simulation is necessary, increasing the computation time. Optimized values of $P=6 \cdot 10^{17} \mathrm{~cm}^{-3}$, $N=4 \cdot 10^{17} \mathrm{~cm}^{-3}$ and $P_{\text {middle }}=3 \cdot 10^{17} \mathrm{~cm}^{-3}$ were obtained for the doping concentrations. Efficiency and Loss as a function of the applied voltage are represented in Fig. 5 for both the model and the complete simulation. Agreement was found in both results. The simplified model reported $V_{\pi} L_{\pi}=1.78 \mathrm{Vcm}$ and Loss $=2.4 \mathrm{~dB}$ for an applied voltage of $3 \mathrm{~V}$, while the complete simulation provided $V_{\pi} L_{\pi}=1.37 \mathrm{Vcm}$ and Loss $=2.2 \mathrm{~dB}$. A relative error below $30 \%$ was achieved using the simplified model. The calculated expected bandwidth was $20 \mathrm{GHz}$.
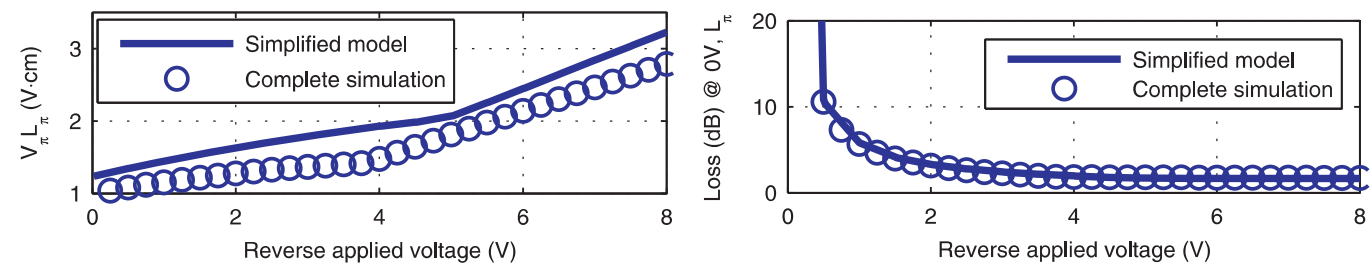

Figure 5. Performance of the PIPIN junction based modulator.

\subsection{High efficient $\mathrm{PN}$ interdigitated junction based modulator}

In order to further increase the efficiency of depletion based modulators PN interdigitated junctions are employed. It consists on periodically introducing PN junctions so that the overlap between the mode and the depletion zone is maximized. It is represented in Fig. 6, the 50nm slab height waveguide was used to further increase the efficiency. It is worth mentioning that given the $3 \mathrm{D}$ nature of the problem, modelling it is challenging.

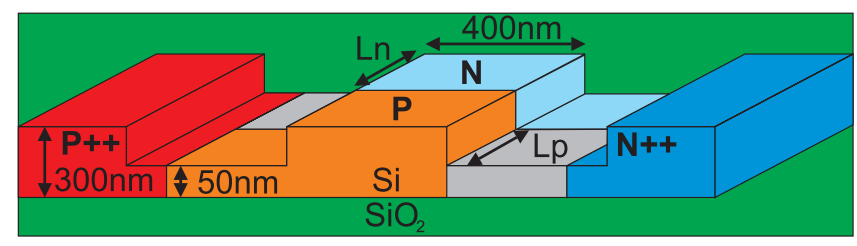

Figure 6. Schematic view of the PN interdigitated junction based modulator.

The simplified model was employed to optimize doping concentrations and fingers lengths. Around 35000 set of values were swept in this case maintaining the computation time below 5 hours. This was possible because the PN junctions were modelled analytically. The obtained values were $P=N=8 \cdot 10^{17} \mathrm{~cm}^{-3}$ and $L_{P}=L_{N}=200 \mathrm{~nm}$ for doping concentrations and fingers length respectively. A complete simulation was carried out for this set of values, taking around 30 hours of computing, mainly because of the 3D electrical simulation. Results of the complete simulation, along with simplified model, are represented in Fig. 7. There is good agreement between them, however a small bias can be observed in the efficiency. The reported values of the simplified model for efficiency and loss at $3 \mathrm{~V}$ were $V_{\pi} L_{\pi}=0.61 \mathrm{Vcm}$ and Loss $=2.7 \mathrm{~dB}$, while complete simulation reported $V_{\pi} L_{\pi}=0.87 \mathrm{Vcm}$ and Loss $=3.9 \mathrm{~dB}$. It is important to point out that in this case the reduction in computation time is over 100 times. The calculated relative error was below $35 \%$, representing a low error given the complexity of the problem and the reduction in computation time. The calculated bandwidth was $10 \mathrm{GHz}$.
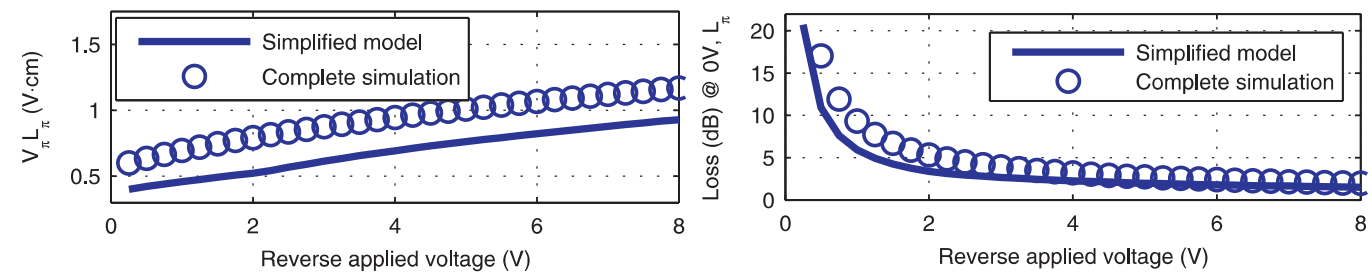

Figure 7. Performance of the PN interdigitated junction based modulator. 


\subsection{Ultra-high efficient PIN injection based modulator}

In order to obtain ultra-high efficiency operation, the PIN injection based modulator is used. It consists on PIN junction which is direct biased in order to inject carriers into the intrinsic zone located in the core of the waveguide, as represented in Fig. 8. However this kind of modulator exhibits a reduce bandwidth because the $\mathrm{RC}$ constant is no longer the limitation but the diffusion-recombination of carriers.

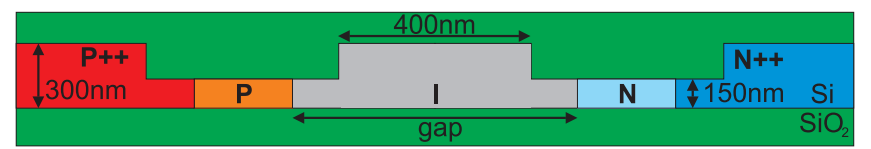

Figure 8. Schematic view of the PIN junction based modulator.

The width of the intrinsic zone (gap) and the doping concentrations were optimized using the simplified model. The swept covered around 1500 set of values and it lasted less than 6 hours. This long computation time is explained because two 1D electrical simulation should be carried out for each set of values, one static simulation to compute efficiency and loss and one transient simulation to compute the bandwidth. Optimized values of $P=N=10^{18} \mathrm{~cm}^{-3}$ and gap $=600 \mathrm{~nm}$ were obtained for doping concentration and width of the intrinsic zone. In this case the complete simulation with one set of parameters took nearly 30 minutes and its results are plotted in Fig. 5, along with the simplified model results. Good agreement can be seen between them. Efficiency and loss values of $V_{\pi} L_{\pi}=0.029 \mathrm{Vcm}$ and Loss $=1.56 \mathrm{~dB}$ were obtained at $1 \mathrm{~V}$ for the simplified model, while the complete simulation reported $V_{\pi} L_{\pi}=0.023 \mathrm{Vcm}$ and Loss $=1.57 \mathrm{~dB}$. Less than $35 \%$ relative error was achieved. The bandwidth was calculated by means of a transient simulation using a square signal as excitation. The calculated bandwidth from rise and fall times was $43 \mathrm{MHz}$.
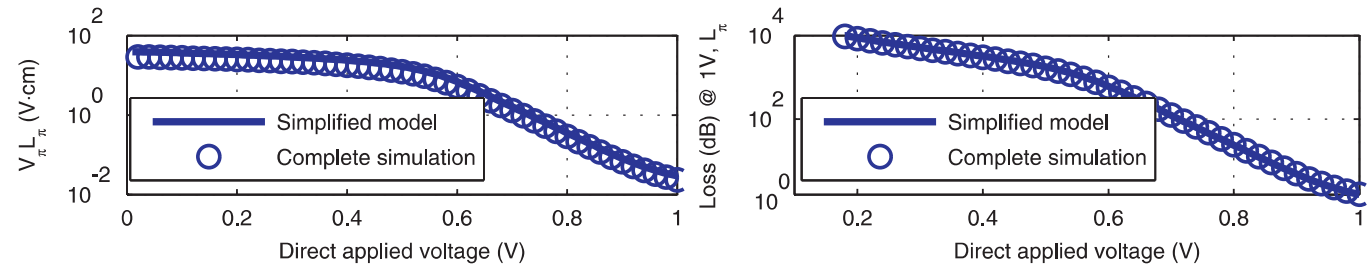

Figure 9. Performance of the PIN junction based modulator.

\subsection{Discussion}

The results presented demonstrate how the proposed simplified model is a powerful tool in the design of silicon phase modulators. The obtained accuracy is sufficient to enable rapid design iteration. Furthermore, the simplified model helped to understand the effect of the different modulator parameters in the overall performance. In general terms it is possible to state that the PIN injection based modulators should be used for applications where efficiency is the most important parameter and bandwidth is not a demand. The PN interdigitated junction based modulators are the best suited for applications where a compromise between bandwidth and efficiency is needed. For low loss applications the PIPIN junction based modulators are the best recommendation. Finally, the PN junction based modulators are the right ones for high bandwidth operation.

\section{CONCLUSION}

The ability to correctly choose the right modulator for the targeted application is very important. In order to do that it is necessary to understand the performance tradeoffs in the choose and design of a silicon phase modulator. Simplified models play a key role in the design stage and are a valuable tool to provide this understanding. A general simplified model has been presented and validated against complete simulations. Furthermore, using the simplified model four different modulators have been optimized and compared. 


\section{Acknowledgements}

This work was supported by the European Plat4m project (FP7-2012-318178) and the European project Cosmicc (H2020-ICT-27-2015-688516). We acknowledge the support of the French Industry Ministry/DGE, through the Nano2017 program.

\section{REFERENCES}

[1] Liow, T.-Y., Song, J., Tu, X., Lim, A.-J., Fang, Q., Duan, N., Yu, M., and Lo, G.-Q., "Silicon optical interconnect device technologies for $40 \mathrm{gb} / \mathrm{s}$ and beyond," Selected Topics in Quantum Electronics, IEEE Journal of 19, 8200312-8200312 (March 2013).

[2] Reed, G. T., Mashanovich, G. Z., Gardes, F. Y., Nedeljkovic, M., Hu, Y., Thomson, D. J., Li, K., Wilson, P. R., Chen, S.-W., and Hsu, S. S., "Recent breakthroughs in carrier depletion based silicon optical modulators," Nanophotonics 3(4-5), 229-245 (2014).

[3] Yang, F., He, Y., Chen, W., and Zhan, Y., "Laser altimeter based on random code phase modulation and heterodyne detection," Photonics Technology Letters, IEEE 26, 2337-2340 (Dec 2014).

[4] Baba, T., Akiyama, S., Imai, M., Hirayama, N., Takahashi, H., Noguchi, Y., Horikawa, T., and Usuki, T., "50-gb/s ring-resonator-based silicon modulator," Opt. Express 21, 11869-11876 (May 2013).

[5] Kajikawa, K., Tabei, T., and Sunami, H., "An infrared silicon optical modulator of metal-oxidesemiconductor capacitor based on accumulation-carrier absorption," Japanese Journal of Applied Physics 48(4), 04C107 (2009).

[6] Marris-Morini, D., Virot, L., Baudot, C., Fédéli, J.-M., Rasigade, G., Perez-Galacho, D., Hartmann, J.-M., Olivier, S., Brindel, P., Crozat, P., et al., "A 40 gbit/s optical link on a 300-mm silicon platform," Optics express 22(6), 6674-6679 (2014).

[7] Ando, T., "Optical coherent beam control based on microwave photonics technologies," in [Microwave Photonics (MWP) and the 2014 9th Asia-Pacific Microwave Photonics Conference (APMP), 2014 International Topical Meeting on], 381-384, IEEE (2014).

[8] Ma, P., Lü, Y., Zhou, P., Wang, X., Ma, Y., and Liu, Z., "Investigation of the influence of mode-mismatch errors on active coherent polarization beam combining system," Optics express 22(22), 27321-27338 (2014).

[9] Soref, R. A. and Bennett, B., "Electrooptical effects in silicon," Quantum Electronics, IEEE Journal of 23, 123-129 (Jan 1987).

[10] Weber, W., McCarthy, S., and Ford, G., "Perturbation theory applied to gain or loss in an optical waveguide," Applied optics 13(4), 715_1-716 (1974).

[11] Jüngel, A., "Drift-diffusion equations," in [Transport Equations for Semiconductors], 1-29, Springer (2009).

[12] http://www.lumerical.com.

[13] Gardes, F., Brimont, A., Sanchis, P., Rasigade, G., Marris-Morini, D., O'Faolain, L., Dong, F., Fedeli, J., Dumon, P., Vivien, L., et al., "High-speed modulation of a compact silicon ring resonator based on a reverse-biased pn diode," Optics express 17(24), 21986-21991 (2009).

[14] Xiao, X., Xu, H., Li, X., Li, Z., Chu, T., Yu, Y., and Yu, J., "High-speed, low-loss silicon mach-zehnder modulators with doping optimization," Optics express 21(4), 4116-4125 (2013).

[15] Ziebell, M., Marris-Morini, D., Rasigade, G., Fédéli, J.-M., Crozat, P., Cassan, E., Bouville, D., and Vivien, L., "40 gbit/s low-loss silicon optical modulator based on a pipin diode," Optics express 20(10), 10591-10596 (2012).

[16] Marris-Morini, D., Baudot, C., Fédéli, J.-M., Rasigade, G., Vulliet, N., Souhaité, A., Ziebell, M., Rivallin, P., Olivier, S., Crozat, P., Roux, X. L., Bouville, D., Menezo, S., Bøuf, F., and Vivien, L., "Low loss $40 \mathrm{gbit} / \mathrm{s}$ silicon modulator based on interleaved junctions and fabricated on $300 \mathrm{~mm}$ soi wafers," Opt. Express 21, 22471-22475 (Sep 2013).

[17] Xiao, X., Xu, H., Li, X., Hu, Y., Xiong, K., Li, Z., Chu, T., Yu, Y., and Yu, J., "25 gbit/s silicon microring modulator based on misalignment-tolerant interleaved pn junctions," Optics express 20(3), 25072515 (2012).

[18] Xu, Q., Manipatruni, S., Schmidt, B., Shakya, J., and Lipson, M., "12.5 gbit/s carrier-injection-based silicon micro-ring silicon modulators," Optics express 15(2), 430-436 (2007). 